\title{
Gene Therapy for Diabetic Retinopathy in Animal Models and Humans
}

\author{
Hiroaki Mizukami*, Masashi Urabe, Akihiro Kume and Keiya Ozawa \\ Division of Genetic Therapeutics, Center for Molecular Medicine, Jichi Medical University, 3311-1 Yakushiji, \\ Shimotsuke, Tochigi, 329-0498, Japan
}

\begin{abstract}
Gene therapy is considered as one of the innovative treatment modalities for diabetic retinopathy (DR). Since genuine animal models of DR are limited, only a few studies have reported the efficacy of gene therapy. For preclinical study of DR, spontaneously diabetic Torii (SDT) rat is a valuable model. Fortunately, we could evaluate the efficacy of adeno-associated virus (AAV)-mediated gene therapy in SDT rats and proved that sFlt-1 expression prevented DR progression. Because of a limited number of large-animal models of DR, it is uncertain whether gene therapy experiments using dogs or monkeys allow reliable conclusions. On the other hand, owing to the recent progress in AAV-mediated gene therapy for retinal diseases in monkeys and humans, gene therapy for DR using AAV vectors may become a reality in the near future.
\end{abstract}

Keywords: Diabetic retinopathy, SDT rat, gene therapy, AAV vector.

\section{CURRENT TREATMENT FOR DIABETIC RETINO- PATHY (DR) AND STATUS OF GENE THERAPY}

Diabetic retinopathy (DR) is usually a consequence of chronic diabetes, often accelerated by poorly controlled disease conditions. It is one of the major causes of blindness in adults, and the number of patients with diabetes is constantly growing. Current standard treatment for DR involves photocoagulation of abnormal vessels in the retina and surgical interventions, along with the control of the underlying diabetes mellitus (DM). Nonetheless, if the disease reaches the proliferative stage, no effective treatments exist to inhibit the progression of DR, which ultimately leads to blindness. Under these circumstances, innovative treatment modalities are required. Gene therapy is regarded as one of these approaches.

Numerous successes, along with side effects, have been reported for gene therapy, showing gradual development of related technologies and their spread into the field of medicine. It is time to apply these technologies to treat larger patient populations and exhibit their importance in medical practice. The efficacy and safety of gene therapy have been proven in many ways. Recently, remarkable results have been achieved by using gene transfer techniques for retinal diseases, which will be discussed in the next section.

A prototype gene therapy strategy is used to transfer gene(s) into target cells and/or tissues, thus enabling the supply of the transgene product locally or systemically for a long period. With increase in the understanding of the pathophysiology and mechanism of DR, different treatment strategies have been postulated as effective interventions for controlling disease progression. Currently, abnormal neovascularization in the retina is the primary target for DR control.

*Address correspondence to this author at the Division of Genetic Therapeutics, Center for Molecular Medicine, Jichi Medical University, 3311-1 Yakushiji, Shimotsuke, Tochigi, 329-0498, Japan; Tel: +81-285-587402; Fax:+81-285-44-8675; E-mail: miz@jichi.ac.jp

\section{TREATMENT STRATEGIES FOR RETINAL DISEASES}

In the quest of developing effective treatments, various vectors have been tested. For the purpose of retinal transduction, adeno-associated virus (AAV) vector is one of the most promising vectors at present. AAV vectors are widely used in clinical trials targeting neurological diseases. One leading example is the Parkinson's disease, for which 3 different clinical trial protocols using AAV-based vectors are successful in phase I [1-4]. The next step will be a comparison between these protocols. Among retinal disorders, Leber's congenital amaurosis (LCA) is currently one of the hottest topics. This disease is a form of retinitis pigmentosa, caused by a mutation in the RPE65 gene. Lack of this gene product results in a deficiency of 11-cis-retinal synthesis, which leads to visual impairment and ultimately loss of vision. Gene therapy approach to correct this deficiency is expected to treat the disease, and success has been achieved in both murine and canine models [5]. Subsequently, human clinical trials have been carried out and successful results have recently been reported [6]. A phase III study is expected to launch in 2011. Another example of success in gene therapy is the correction of color blindness in monkeys by transferring of the L-opsin gene into the retina using AAV5-based vectors [7]. These reports should make human clinical trials of DR into reality.

While designing the strategy of gene therapy, the choice of the transgene is critical. Pathophysiology of DR has been well analyzed, and factors responsible for the disease have been extensively reviewed [8]. Among the possible factors, vascular endothelial growth factor (VEGF) plays the most crucial role in developing the disease conditions.

One example is age-related macular degeneration (AMD), especially wet type; this disease is closely related to DR in that pathological neovascularization mediated by local VEGF is the principal feature. Therefore, therapeutic approaches for AMD should be applied to DR in principle. Currently, intraocular injection of antibody against VEGF 
(ranibizumab, or bevacizumab) has become a common practice to control wet AMD (reviewed in [9]). This treatment modality is effective soon after injection, and gradually deteriorates over time. Monthly administration is considered as the standard treatment; however, each time the procedure involves ocular penetration with some risk of infection or other complications. To extend the duration of therapeutic efficacy and minimize the risk and pain associated with intraocular administration, gene-mediated therapy may be the ultimate solution.

To suppress excessive VEGF production, a number of approaches are known. One of the most well-known method is the expression of soluble VEGF receptor, sFlt-1 [10]. The efficacy of this strategy using adenoviral and AAV vectors has been demonstrated in mice $[11,12]$ and subsequently, in larger animal models. Adenoviral vectors have commonly been used in earlier studies; however, the duration of expression tends to be shorter even with helper-dependent adenoviral vectors [13]. A different approach is the expression of pigment epithelium-derived factor (PEDF), which also exhibits anti-angiogenic actions. A clinical trial using adenoviral vector (AdPEDF) was conducted safely [14]. Other approaches include the utilization of aptamers and siRNA. As for the aptamers, one of the results of a clinical trial using pegaptanib was reported [15]. In order to increase the efficiency of the delivery and/or stability of siRNA, numerous attempts have been made. In any case, a number of treatment conditions, e.g., route of administration, volume of injection, choice of serotypes and promoters, dose of the vector, etc, need to be explored to obtain maximum therapeutic efficacy while avoiding potential risk of VEGF oversuppression, which may result in retinal atrophy [16] or threaten vascular maintenance [17].

In designing the therapeutic strategy using AAV vectors, selection of the vector serotype becomes an important issue. To date, numerous serotypes or clones of AAV have been developed as vectors. Among them, AAV2 and AAV5 are most commonly used for retinal transduction. In studies targeting the central nervous system, AAV2 shows specificity to neuronal cells, whereas AAV5 transduces both neurons and glial cells [18]. Similarly, in retinal transduction, neuron-specificity was observed in AAV2, whereas AAV5-based vector has shown less-specific, generalized transduction; in experiments targeting the primate retina, preferential transduction of rods has also been observed using AAV5 [19]. In order to suppress retinal neovascularization, both AAV2 and AAV5 work fine and it has not been determined which is better. Although other serotypes or clones have also been used, there is no clear evidence as to whether they have any advantage over the classic serotypes in retinal transduction and suppression of abnormal neovascularization.

With regard to the promoters, cytomegalovirus (CMV) promoter is most commonly used, at least in the case of AAV vectors, especially when neuronal cells are transduced. In general, it is ideal to use tissue-specific promoters to achieve better therapeutic outcomes and avoid inadvertent gene expression. In the case of retinal transduction, promoters specific for rods or cones may be used [20]. However, tissue-specific promoters are considerably weaker than CMV or other virus-derived promoters, and a much higher vector dose may be necessary to achieve the desired outcome.

The method of intraocular administration is also a matter of discussion. Subretinal injection is the standard method for most vector systems including AAV vector. In fact, in the recent clinical trials and monkey experiments, vectors have been administered subretinally [6, 7]. This method requires expertise and is accompanied by the risk of visual impairment, especially when the region of macula or its vicinity is transduced. Moreover, the volume of injection is relentlessly restricted. Thus, intravitreal injection is an appropriate method to overcome these limitations. However, simple intravitreal injection usually results in inefficient retinal transduction and conquering this problem is essential to effectively promote clinical trials with safety. Attempts have been made for this purpose, and a recent report showed improved efficiency by including pronase at a minimum concentration to destroy the outer membrane and enhance retinal transduction [21].

\section{MODELS OF DR OTHER THAN SPONTA- NEOUSLY DIABETIC TORII (SDT) RAT}

It is surprising that there are only limited numbers of DR models with 'genuine' diabetic status. Most of the "retinopathy" models are obtained through exposure to various levels of oxygen tensions. This method is already well established and widely used in neovascularization research [22]. At the same time, the extent to which these models reproduce real conditions of human DR remains arguable. The actual reasons why most animals with diabetes do not show recognizable DR during their life span need to be clarified. It seems that the maturation of $\mathrm{DR}$ requires constant diabetic conditions for a certain period of time. In rodents, no DR models can be recognized other than the SDT rat.

Even in large animals, authentic DR models are scarce. A report indicates DR-like changes in galactose-fed dogs [23]. This model required 7 years to develop the changes, which indicates that it is not easy to follow the experiments conducted and utilize the animals for further experiment. In fact, there are only a few reports on studies utilizing this model. Interestingly, the same model showed DR progression even after the reversal of diabetic conditions by discontinuation of galactose administration [24]. In monkeys, DR status has been reported in those who developed diabetes spontaneously [25]. This study included 16 animals; the DR status was related to the duration of diabetes, since 1 animal with less than 1 year of diabetes did not show DR, while the remaining animals that developed DR shared a history of diabetes for 6 years or longer. The necessity of chronic diabetic conditions for DR development and progression is similar for humans. In one study, a unique monkey model was developed by intraocular injection of AAV vectors encoding VEGF [26]. In groups receiving subretinal injection, retinal neovascularization emulating DR was observed. Further, after intravitreal injection of the vector, neovascularization was observed in the anterior portion of the eye, i.e., the cornea and iris. Thus, this study precisely reflected the intraocular transduction pattern of AAV vectors after intravitreal injection to the monkey model. Although this model is potentially useful in the study 
of DR, it may not be suitable for the assessment of AAV vector-mediated gene therapy since the treatment involves readministration of AAV vector with immunological consequences.

\section{UTILITY OF SDT RAT AS A MODEL OF DR}

As discussed thoroughly in this issue, SDT rat is a valuable model of DR [27], mimicking human disease conditions [28]. Fortunately, we could perform experiments investigating the efficacy of gene therapy by using SDT rats [29]. For these experiments, our earlier experiments using sflt-1-mediated suppression of VEGF [30] served as the platform. While designing the study, one of the biggest issues before us was to ascertain the exact goal of the experiments, i.e., to determine whether the method had therapeutic potential or exerted preventive effects. Therapeutic experiment seems more important and valuable. However, its conduction is actually quite difficult. For

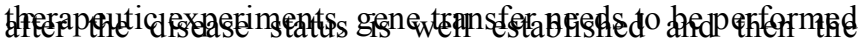

efficacy can be proved by showing improved disease conditions through monitoring of the animals. In this case, several problems have been put forward. The most fundamental question is whether the disease status of DR can be reversed by gene therapy alone. Another difficulty lies in monitoring retinal conditions sequentially; precise evaluation of the retina can be performed only by fluorescein angiography (FAG), which is usually performed at the time of sacrifice. Under these circumstances, we aimed to demonstrate the preventive effects rather than the therapeutic outcome. To demonstrate the preventive effects, sflt-1 gene was transduced unilaterally before the DR was developed, and its expression was examined at the time of DR maturation. Since immunohistochemical detection of the transgene product (sFlt-1) was not stable at the time of initiation, each eye received an additional AAV vector carrying the lacZ gene, and distribution of the vector was estimated by staining of the retinal tissue for X-gal. At the time of analysis, no prior information about the treatment regimen was available to keep the judgments unbiased. Finally, the overall results indicated that the side injected with the sflt-1-carrying vector clearly showed suppression of DR [29].

Although a long waiting period is required between the time of injection and analysis, the study progressed constantly since the SDT rats exhibited reasonably high rate of DR at the time of analysis (60 weeks of age). At the time of maturation of DR, the animals became vulnerable to stresses caused by transportation, change in the atmosphere, etc. Therefore, special care should be taken to decrease the stresses to the animals around the time of analysis.

In conclusion, SDT rat is suitable for experiments to evaluate the preventive effects of therapeutic modalities such as gene therapy. However, it remains unclear whether even with this model, therapeutic experiments can successfully be achieved because of the reasons stated earlier.

\section{FUTURE DIRECTIONS-TOWARD GENE THERAPY FOR DR IN HUMANS}

In the development of novel therapeutic approaches, once the efficacy of the therapy is established in experiments using rodents, the next step is conduction of experiments with larger animals, e.g., dogs or monkeys, before proceeding to human clinical trials. However, in the case of DR, this may not be true. First, animal models of genuine DR are seriously limited. If we use only genuine DR models, decades will be passed to accomplish the experiment using dogs or monkeys. Second, remarkable success has been achieved in gene therapy for retinal diseases. Experiences in gene therapy for retinal diseases, especially LCA and AMD, should support gene therapy for DR. Since the safety of gene therapy using AAV has been warranted in every clinical trial, it is likely that after proving the efficacy in monkey models of neovascularization, human clinical trials will be initiated. Gene therapy may be a treatment of choice for DR in the near future.

\section{REFERENCES}

[1] Eberling JL, Jagust WJ, Christine CW, et al. Results from a phase I safety trial of hAADC gene therapy for Parkinson disease. Neurology 2008; 70: 1980-3.

[2] Kaplitt MG, Feigin A, Tang C, et al. Safety and tolerability of gene therapy with an adeno-associated virus (AAV) borne GAD gene for Parkinson's disease: an open label, phase I trial. Lancet 2007; 369: 2097-105.

[3] Marks WJ, Jr., Ostrem JL, Verhagen L, et al. Safety and tolerability of intraputaminal delivery of CERE-120 (adenoassociated virus serotype 2-neurturin) to patients with idiopathic Parkinson's disease: an open-label, phase I trial. Lancet Neurol 2008; 7: 400-8.

[4] Muramatsu S, Fujimoto K, Kato S, et al. A phase I study of aromatic L-amino acid decarboxylase gene therapy for Parkinson's disease. Mol Ther 2010; 18: 1731-5.

[5] Bennicelli J, Wright JF, Komaromy A, et al. Reversal of blindness in animal models of leber congenital amaurosis using optimized AAV2-mediated gene transfer. Mol Ther 2008; 16: 458-65.

[6] Maguire AM, High KA, Auricchio A, et al. Age-dependent effects of RPE65 gene therapy for Leber's congenital amaurosis: a phase 1 dose-escalation trial. Lancet 2009; 374: 1597-605.

[7] Mancuso K, Hauswirth WW, Li Q, et al. Gene therapy for redgreen colour blindness in adult primates. Nature 2009; 461: 784-7.

[8] Ting JH, Martin DK. Basic and clinical aspects of gene therapy for retinopathy induced by diabetes. Curr Gene Ther 2006; 6: 193-214.

[9] Bressler SB. Introduction: Understanding the role of angiogenesis and antiangiogenic agents in age-related macular degeneration. Ophthalmology 2009; 116: S1-7.

[10] Kendall RL, Thomas KA. Inhibition of vascular endothelial cell growth factor activity by an endogenously encoded soluble receptor. Proc Natl Acad Sci U S A 1993; 90: 10705-9.

[11] Bainbridge JW, Mistry A, De Alwis M, et al. Inhibition of retinal neovascularisation by gene transfer of soluble VEGF receptor sFlt1. Gene Ther 2002; 9: 320-6.

[12] Honda $M$, Sakamoto $T$, Ishibashi $T$, Inomata $H$, Ueno $H$. Experimental subretinal neovascularization is inhibited by adenovirus-mediated soluble VEGF/flt-1 receptor gene transfection: a role of VEGF and possible treatment for SRN in age-related macular degeneration. Gene Ther 2000; 7: 978-85.

[13] Lamartina S, Cimino M, Roscilli G, et al. Helper-dependent adenovirus for the gene therapy of proliferative retinopathies: stable gene transfer, regulated gene expression and therapeutic efficacy. J Gene Med 2007; 9: 862-74.

[14] Campochiaro PA, Nguyen QD, Shah SM, et al. Adenoviral vectordelivered pigment epithelium-derived factor for neovascular agerelated macular degeneration: results of a phase I clinical trial. Hum Gene Ther 2006; 17: 167-76.

[15] Gragoudas ES, Adamis AP, Cunningham ET, Jr., Feinsod M, Guyer DR. Pegaptanib for neovascular age-related macular degeneration. N Engl J Med 2004; 351: 2805-16.

[16] Murakami Y, Ikeda Y, Yonemitsu Y, et al. Inhibition of choroidal neovascularization via brief subretinal exposure to a newly developed lentiviral vector pseudotyped with Sendai viral envelope proteins. Hum Gene Ther 2010; 21: 199-209.

[17] Lee S, Chen TT, Barber CL, et al. Autocrine VEGF signaling is required for vascular homeostasis. Cell 2007; 130: 691-703. 
[18] Tenenbaum L, Chtarto A, Lehtonen E, Velu T, Brotchi J, Levivier M. Recombinant AAV-mediated gene delivery to the central nervous system. J Gene Med 2004; 6(Suppl 1): S212-22.

[19] Lotery AJ, Yang GS, Mullins RF, et al. Adeno-associated virus type 5: transduction efficiency and cell-type specificity in the primate retina. Hum Gene Ther 2003; 14: 1663-71.

[20] Semple-Rowland SL, Coggin WE, Geesey M, et al. Expression characteristics of dual-promoter lentiviral vectors targeting retinal photoreceptors and Muller cells. Mol Vis 2010; 16: 916-34.

[21] Dalkara D, Kolstad KD, Caporale $\mathrm{N}$, et al. Inner limiting membrane barriers to AAV-mediated retinal transduction from the vitreous. Mol Ther 2009; 17: 2096-102.

[22] Connor KM, Krah NM, Dennison RJ, et al. Quantification of oxygen-induced retinopathy in the mouse: a model of vessel loss, vessel regrowth and pathological angiogenesis. Nat Protoc 2009; 4: 1565-73.

[23] Kador PF, Takahashi Y, Wyman M, Ferris F, 3rd. Diabeteslike proliferative retinal changes in galactose-fed dogs. Arch Ophthalmol 1995; 113: 352-4.

[24] Engerman RL, Kern TS. Retinopathy in galactosemic dogs continues to progress after cessation of galactosemia. Arch Ophthalmol 1995; 113: 355-8.
[25] Kim SY, Johnson MA, McLeod DS, et al. Retinopathy in monkeys with spontaneous type 2 diabetes. Invest Ophthalmol Vis Sci 2004; 45: 4543-53.

[26] Lebherz C, Maguire AM, Auricchio A, et al. Nonhuman primate models for diabetic ocular neovascularization using AAV2mediated overexpression of vascular endothelial growth factor. Diabetes 2005; 54: 1141-9.

[27] Shinohara M, Masuyama T, Shoda T, et al. A new spontaneously diabetic non-obese Torii rat strain with severe ocular complications. Int J Exp Diabetes Res 2000; 1: 89-100.

[28] Kakehashi A, Saito Y, Mori K, et al. Characteristics of diabetic retinopathy in SDT rats. Diabetes Metab Res Rev 2006; 22: 45561.

[29] Ideno J, Mizukami H, Kakehashi A, et al. Prevention of diabetic retinopathy by intraocular soluble flt-1 gene transfer in a spontaneously diabetic rat model. Int J Mol Med 2007; 19: 75-9.

[30] Hasumi Y, Mizukami H, Urabe M, et al. Soluble FLT-1 expression suppresses carcinomatous ascites in nude mice bearing ovarian cancer. Cancer Res 2002; 62: 2019-23.

(C) Mizukami et al.; Licensee Bentham Open.

This is an open access article licensed under the terms of the Creative Commons Attribution Non-Commercial License (http://creativecommons.org/licenses/by-nc/3.0/) which permits unrestricted, non-commercial use, distribution and reproduction in any medium, provided the work is properly cited. 